\title{
In vitro antimicrobial activity of ethanol and water extracts of Cassia alata
}

\begin{abstract}
Crude ethanol and water extract of leaves and barks from Cassia alata were tested in vitro against fungi, (Aspergillus fumigatus and Microsporum canis), yeast (Candida albicans) and bacteria (Staphylococcus aereus and Escherichia coli). C. albicans showed concentrationdependent susceptibility towards both the ethanol and water extracts from the barks, but resistant towards the extracts of leaves. The degree of susceptibility varied, the water extract from barks showed bigger inhibition zone than the ethanol extracts (12ï 16 and $10 \ddot{1} 14 \mathrm{~mm}$, diameter respectively). The growth of Aspergillus fumigatus and Microsporum canis were not affected by all types of the plant extracts. Results were comparable to standard antifungal drug Tioconazole (18 $\mathrm{mm}$ diameter) at equivalent concentration. The anti-bacterial activity of C. alata extracts on S. aureus was detected with only the leaves extracts using water and ethanol. The water extract exhibited higher antibacterial activity than the ethanol extract from leaves (inhibition zones of $11 \mathrm{ï} 14$ and $9 \ddot{1} 11 \mathrm{~mm}$, respectively). E. coli showed resistance to all types of extracts. Based on the current findings, it can be concluded that this plant has antimicrobial activity, which is as potent as standard antimicrobial drugs against certain microorganisms.
\end{abstract}

Keyword: Cassia alata; Antifungal; Antibacterial; In vitro 